\title{
AMPLITUDE NOISE INDUCED PHASE NOISE IN ELECTROSTATIC MEMS RESONATORS
}

\author{
Manu Agarwal, Kwan K. Park, Bongsang Kim, Matthew A. Hopcroft, Saurabh A. Chandorkar, \\ Rob N. Candler ${ }^{1}$, Chandra M. Jha, Renata Melamud, Thomas W. Kenny, Boris Murmann \\ Departments of Electrical and Mechanical Engineering, Stanford University, \\ Stanford, California USA
}

\begin{abstract}
This work investigates the effects of amplitude perturbations on the frequency stability of Electrostatic MEMS resonators. As in quartz, the activity limit, or maximum sustainable drive current depends on the nonlinearities of the resonator and its quality factor $(Q)$. We find that at high activity the random amplitude fluctuations get mixed into the phase noise of the oscillator, causing frequency instability (phase noise). Using a DoubleEnded-Tuning-Fork (DETF) structure we verify the presence of $A-f$ (Amplitude frequency dependence) effect in MEMS resonators. We find that the $A-f$ coefficient in our low frequency test structure is orders of magnitude higher when compared to quartz crystal resonators.
\end{abstract}

\section{INTRODUCTION}

Electrostatically coupled micromechanical devices have become a promising solution for frequency references and signal processing applications in recent times [1]. Integration of these micromechanical structures with on-chip CMOS circuitry potentially leads to considerable size and cost reduction, making them a commercially attractive and viable replacement for quartz crystal technology.

Several efforts to commercialize electrostatic MEMS resonator technology are currently underway. One of the most promising applications of commercial interest is probably the cellular communication business. MEMS resonator based oscillators with frequency stability that satisfies the GSM standard requirements have already been reported in $[2,3]$. Other important applications include networking and timekeeping. While some of these applications focus on higher frequency resonators, low frequency and flexural type resonators appear in a wide assortment of resonant sensing applications including acceleration sensors, mass sensors, etc.

For these applications, nonlinearities in the resonators limit the ultimate frequency stability (or phase noise performance) that can be achieved. In sensors, this stability is a measure of the achievable resolution. Nonlinearities in quartz have been studied and their impact on frequency stability of oscillators has been discussed [4]. Perhaps the most important limitation on frequency stability is dictated by the limitation on the 'activity' or power handling, which is a measure of the useful signal strength that can be sustained by these devices. At high amplitudes of oscillation the nonlinearities can become considerable and cause Duffing type effects. The broader term used for this effect in quartz literature is $A-f$ (Amplitude-frequency) effect. The generating mechanisms for nonlinearities that can cause this effect in MEMS and a mathematical description for power handling have been developed previously [3, 5]. Nonlinear multiplication of low frequency electronics noise into the close-to-carrier noise have also been studied [6-8].

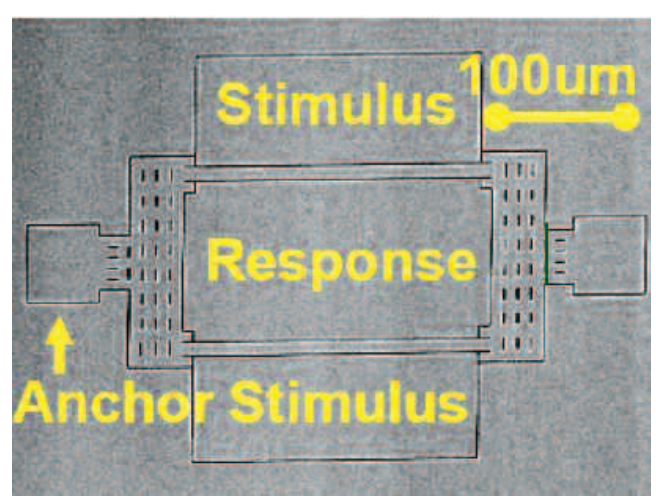

(a)

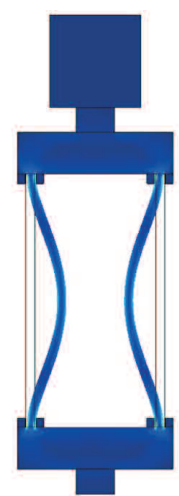

(b)
Figure 1. a) Scanning electron micrograph of the top view of the DETF resonator used in our experiments. The beam dimensions used were $L=220 \mu \mathrm{m}, w=8 \mu \mathrm{m}$ and $t=20 \mu \mathrm{m}$. The resonant frequency of this resonator is $\sim 1.3 \mathrm{MHz}$. b) FEMLAB simulation showing the resonant mode shape of the structure.

In this work we focus primarily on the $A-f$ effect, which converts the amplitude noise into frequency noise, and at higher amplitudes can even cause bifurcation instability [3, 5]. Analytical models for the effect of this nonlinearity on the noise performance of these oscillators will be presented, along with experimental verification using a double-ended tuning fork resonator, schematically shown in Figure 1. Methods for measurement and quantification of nonlinearities and comparison with quartz will also be presented.

\section{AMPLITUDE NOISE vs. PHASE NOISE}

In this section we describe the two kinds of noise in an oscillator output, namely amplitude noise and phase noise. The output voltage of an oscillator can be represented by

$$
V_{\text {out }}(t)=\left(A_{0}+a_{N}(t)\right) \cos \left(2 \pi f_{0} t+\varphi_{N}(t)\right)
$$

Here $A_{0}$ is the average amplitude of the output signal and $f_{0}$ is nominal frequency of oscillation. $a_{N}(t)$ and $\varphi_{N}(t)$ represent the time varying components of the amplitude and phase, respectively. These components are considered as noise, because an ideal oscillator would have constant amplitude $A_{0}$ and phase varying at a constant rate $2 \pi f_{0}$. It can be shown that white noise power of the amplifier electronics is equally partitioned into amplitude and phase noise components [9].

The low frequency noise components, at $f_{N}<<f_{0}$, of $a_{N}(t)$ and $\varphi_{N}(t)$ are most important because they appear at $f_{0} \pm f_{N}$ in the output spectrum of the oscillator, and form the "close to carrier" noise.

In most commercial applications where these devices are used as timing or frequency references, the amplitude noise $a_{N}(t)$ can be ignored. As can be seen from (1), the amplitude noise by itself does not affect the temporal position of the zero crossings of the

\footnotetext{
${ }^{1}$ Current address - BOSCH Research and Technology Centre, Palo Alto, CA
} 
signal where all the useful timing information is situated. Phase noise $\varphi_{N}(t)$, on the other hand, affects only the zero crossings and is hence a measure for the frequency stability of the oscillator. Therefore, it is hence more relevant for timing and frequency reference applications. Additionally, amplitude noise can be removed, if needed, using simple amplitude saturation or hard limiting of the oscillator output to remove amplitude fluctuations, followed by filtering to remove the harmonics. Such a practical method is not available for removing phase noise.

Because MEMS resonators have low intrinsic power handling compared to quartz they almost always need to be operated close to their nonlinear limits $[3,5,6]$. At such high amplitudes the $A-f$ effect becomes even more important. It is hence important to design the sustaining circuit with stable amplitude at the input of the resonator. In most cases this can be achieved by limiting the amplitude of the AC voltage using a feedback loop and a variable gain amplifier (VGA) or hard limiting with something similar to a comparator.

\section{AMPLITUDE-FREQUENCY DEPENDENCE OR $A-f$ EFFECT}

It is widely mentioned in quartz literature that the $A-f$ and related effects limit the usable amplitude of resonator vibration [4, 10], defining the maximum signal strength available. In this section, we give a mathematical description of this effect in MEMS resonators. These models quantitatively describe the nonlinear distortion of the resonator response $[3,5]$, thereby providing an important design objective.

Since the dominant nonlinearities in these devices are of $2^{\text {nd }}$ and $3^{\text {rd }}$ order in the restoring force, the amplitude-frequency relationship will exhibit a parabolic dependence for high $Q$ resonators [11]. Ignoring thermal effects this can be represented by

$$
\frac{\Delta f}{f_{0}}=\kappa I_{d}{ }^{2}
$$

where $I_{d}$ is the r.m.s. drive current through the resonator and $\kappa$ is called the $A-f$ coefficient, consistent with quartz terminology.

MEMS resonators exhibit two kinds of nonlinearities electrical softening and mechanical stiffening [3, 5]. Quartz exhibits only stiffening type nonlinearities. At low DC bias voltages, the capacitive forces are weaker and the dominant nonlinearities occur due to geometrical and material effects at large displacements/strains. This causes mechanically induced stiffening type $A-f$ effect. At higher bias voltages the capacitive forces become stronger and hence the electrostatic nonlinearities become dominant. This causes electrically induced softening $A-f$ effect.

Mechanical nonlinearities are similar to the kind seen in quartz [4], where the resonator response bends toward the higher frequency side. This is caused by mechanical stiffening of the effective spring constant at higher amplitudes. This effect is shown in Figure 2(a). The device used for this measurement was fabricated using the epi-seal encapsulation process as discussed in [12].

We can express the mechanical nonlinearity terms in the system by looking at the higher order components in the mechanical restoring (or spring) force, given by

$$
F_{\text {spring }}=-k_{1} x-k_{3} x^{3}
$$

where $k_{1}$ is the linear spring constant, $k_{3}$ is the $3^{\text {rd }}$ order force nonlinearity component and $x$ is the beam displacement. The $2^{\text {nd }}$ order nonlinearity component has been ignored due to symmetry of the structure. The negative sign here indicates the restoring nature of the force. For this system the mechanical $A-f$ coefficient is given by $[5,11]$

$$
\kappa_{m}=\frac{3 k_{3} d^{4}}{4 m \omega_{0}^{4} \varepsilon^{2} A^{2} V_{\text {Bias }}^{2}}
$$

where $d$ is the electrostatic gap size, $m$ is the effective mass of the structure, $\omega_{0}$ is the angular frequency, $A$ is the area of transduction capacitance, and $V_{\text {Bias }}$ is the DC bias voltage on the structure. This effect may not be observed in devices with low $Q$ or very small gap size. This is because under these conditions the amplitude of oscillation required for getting an amplitude based frequency shift comparable to the bandwidth of the resonator becomes a larger fraction of the electrostatic gap size. The system cannot be modeled with $2^{\text {nd }}$ and $3^{\text {rd }}$ order nonlinearities alone in this case, and higher electrostatic nonlinearities start to have an impact. These electrostatic nonlinearities are always of the softening type, making the mechanical stiffening effect impossible to observe.

Electrical nonlinearities make the effective stiffness of the device smaller at high amplitudes, leading to a resonator response that progressively bends towards the lower frequency side as we increase the drive current. Figure 2(b) illustrates this effect. The nonlinearities in the electrostatic forces are due to the change in the gap size as the resonator vibrates. This can be accurately modeled by looking at the higher order Taylor coefficients of the expression for the force between the plates of a capacitor. Using the third order nonlinearity term, the electrical $A-f$ coefficient becomes $[5,11]$

$$
\kappa_{e}=-\frac{1}{2 m \omega_{0}^{4} \varepsilon A d}
$$

In this regime, $\kappa$ is independent of the bias voltage. This effect should be observed at high bias voltages in almost every electrostatic MEMS resonator. It is also to be noted that the models presented up to this point are not specific to flexural designs but are valid for all types of electrostatic resonators.

\section{STATIC MEASUREMENT OF $\boldsymbol{A}-\boldsymbol{f}$ EFFECT}

To measure the $A-f$ coefficient using this method, we need to measure the frequency shift as a function of the drive current level, and extract the parabolic dependence coefficient. Figure 3 shows the peak frequency plotted against the peak activity for different regimes of operation in the resonators. The inset in this figure shows the distortion in the response as we change the drive level for this resonator. The device in this measurement is the same design as specified before, but fabricated using the oxide seal encapsulation process [13]. The device layer thickness was $20 \mu \mathrm{m}$ with an electrostatic gap size of $\sim 2 \mu \mathrm{m}$.

\section{DYNAMIC MEASUREMENT OF $\boldsymbol{A}$ - $\boldsymbol{f}$ EFFECT}

An alternative way of measuring the $A-f$ coefficient is using dynamic measurements, a technique also used in quartz crystal resonators [14]. In this method we connect the resonator in a self oscillating circuit and modulate the amplitude of the voltage input to the resonator at several test frequencies $\left(f_{N}<<f_{0}\right)$, looking for phase perturbations in the oscillator output.

This technique has several advantages. First, it can be used to isolate the thermally induced frequency shift effect from the nonlinear effect to make a more accurate measurement of the nonlinear properties. Second, since this is a small perturbation experiment, we can verify whether the impact of random amplitude perturbations in the real system can be completely explained from this nonlinear effect. This is so, because these forced small amplitude perturbations emulate the random amplitude perturbations that would be present in a real oscillator more accurately. Finally, since this measurement is done on an oscillator 


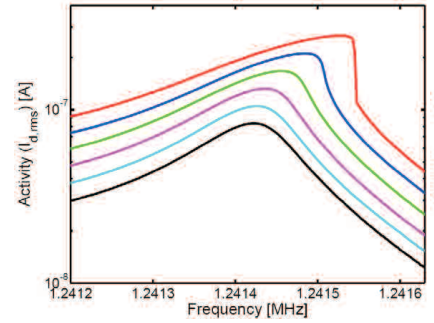

(a)

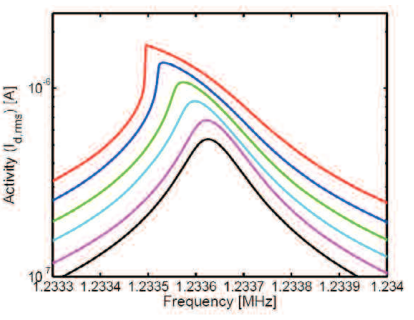

(b)
Figure 2. Measured A-f effect in a DETF MEMS resonator. The plots show activity ( $I_{\text {drive }}$ ) as a function of frequency at a) low bias voltage $(13 \mathrm{~V})$ showing mechanical nonlinearities and b) at high bias voltage $(60 \mathrm{~V})$, showing electrical nonlinearities.

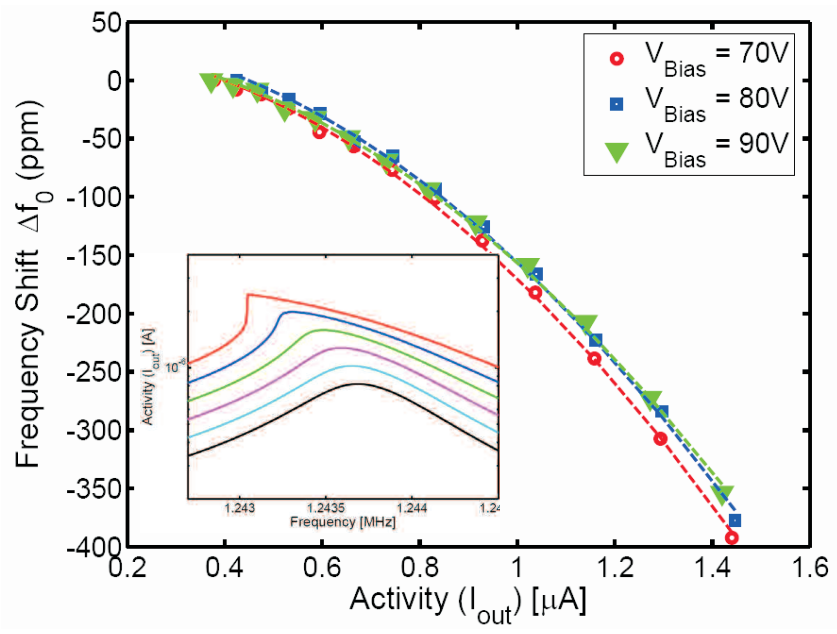

Figure 3. Static measurement of the electrical A-f coefficient. The plot shows the peak frequency as a function of the peak output current at different bias voltages. The dashed lines are parabolic fits. It can be seen that a very good match of dependence is observed with the model developed in equation (2). Also, as predicted by equation (5), we find that the $\kappa$ for the resonator remains independent of bias voltage. The inset shows resonator frequency responses with different activity levels at $V_{\text {Bias }}=70 \mathrm{~V}$.

rather than a resonator, the measurement includes the effects of any circuit non-idealities (like phase error) that may be present. Hence it can be used to provide a figure of merit for the complete oscillator circuit. Figure 4 shows a schematic of the oscillator circuit used for this measurement and a picture of the PCB fabricated for this circuit.

Without amplitude modulation at the input, the output frequency spectrum of the oscillator would be a single peak at the resonant frequency. However, when we introduce amplitude modulation at the input at a frequency $f_{N}$ we see side-peaks at $f_{0} \pm$ $f_{N}$ as shown in Figure 5.

The phase information of the sidepeak is not available in this measurement. This means that the "Amplitude Noise (AN) - Phase Noise (PN)" sidepeak could also be "PN - AN" depending on the relative magnitudes. Hence, in order to extract the phase perturbation component we look at both, the sum and difference of the side-peak levels. A more precise method to do this is to use a phase bridge as described in [14]. An extracted phase perturbation level plot is shown in figure 6. It should be noted that this graph does not plot the inherent random phase noise level of the oscillator. This is the phase perturbation level due to the controlled

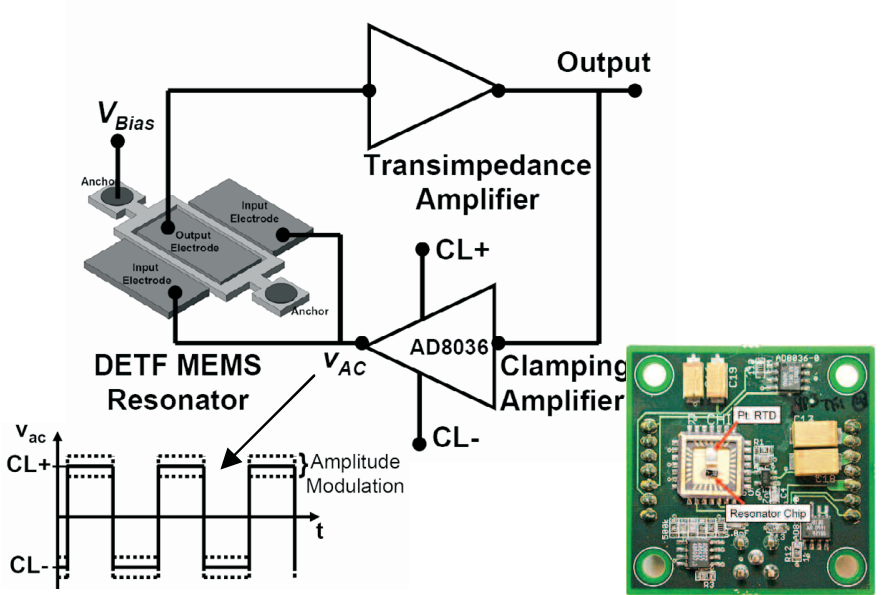

Figure 4. Circuit schematic and $P C B$ of the oscillator. The AD8036 is a clamping amplifier that limits the peak levels of $v_{A C}$ to $C L+$ and $C L-$. Amplitude modulation of the resonator driving current was achieved by superposing a small sinusoidal signal on the $C L+$ and $C L-$ voltage levels using simple bias tees.

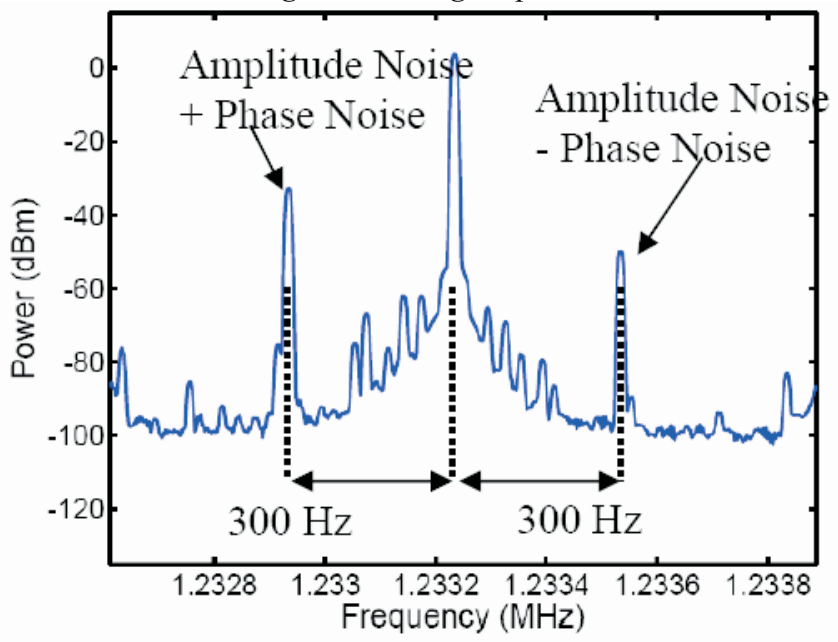

Figure 5. Output spectrum of the oscillator with the resonator input having amplitude modulation at $300 \mathrm{~Hz}$. In this case where $\kappa$ is negative, the low frequency sideband can be shown to be the sum of amplitude and phase perturbations and the higher frequency sideband is the difference of amplitude and phase perturbations. The inequality of sidebands confirms that amplitude perturbations in the input cause phase perturbations, or that amplitude noise is converted to phase noise due to the A-f effect.

amplitude modulation at the input, which is much higher than the inherent random phase noise of the oscillator. Close-to-carrier perturbation exhibits $1 / f^{2}$ behavior which represents a constant frequency perturbation at the modulation frequency. We can estimate the $\kappa$ of the device from the relative strength of the sidepeak level $L\left(f_{m}\right)$ in $d B c$, by

$$
L\left(f_{m}\right)=20 \log \left(\frac{f_{0} \kappa I_{d} \Delta I_{d}}{f_{m}}\right)
$$

where $f_{m}$ is the amplitude modulation frequency. In this equation, if $\Delta I_{d}$ is replaced by the random amplitude noise in the drive current, we will obtain the phase random noise in the system. The extracted value of $\kappa$ from the dynamic measurements and comparison with static measurements are shown in Figure 7. 


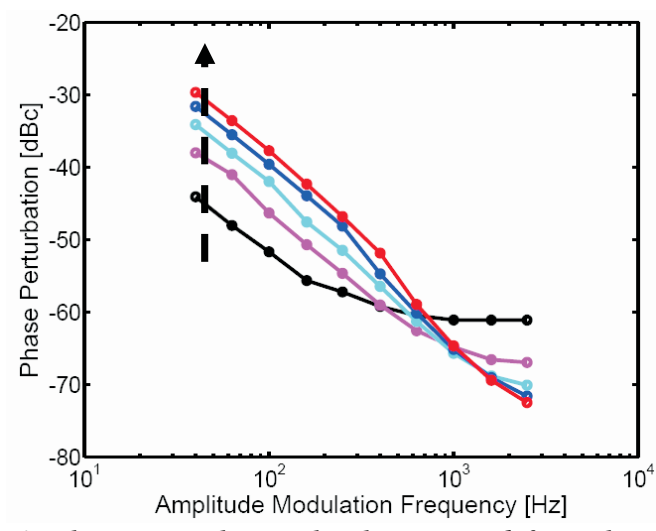

Figure 6. Phase perturbation levels extracted from the sidepeak levels in the output spectrum (Figure. 5). The 1/f behavior close to the carrier can be observed from this plot, consistent with the model, as shown in (6). The different curves represent the phase perturbation levels at different input $A C$ voltages from $0.1 V_{p}$ to $0.5 V_{p}$ with a constant modulation voltage of $9 \mathrm{mV}$ superposed on it. The arrow points in the direction of measurements obtained for increasing input $A C$ voltages.

\section{DISCUSSION}

We found that the $A-f$ coefficient of the DETF MEMS resonator considered here is very large. This is representative of its power handling capability, and such high numbers suggest that the sustainable drive current is merely on the order of $\mu \mathrm{A}$, even with a $\mathrm{Q}$ of the order or $10^{4}$, as found in [5]. By comparison, a $5-\mathrm{MHz}$ quartz AT cut crystal has $\kappa \sim 10^{-13} /(\mu \mathrm{A})^{2}$ [4] which is about 9 orders of magnitude lower than MEMS $\left(\kappa \sim 10^{-4} /(\mu \mathrm{A})^{2}\right)$. Hence, quartz crystals can sustain drive currents of several $\mathrm{mA}$ even though the quality factor is comparatively high, about $10^{6}$.

As can be seen in equations (4) and (5), the $\kappa$ coefficient in MEMS resonators should decrease considerably as we go to higher frequencies $\left(\omega_{0}\right)$. For instance, a $100-\mathrm{MHz}$ resonator built on a similar process could have its $A-f$ coefficient within approximately an order of magnitude of quartz $\left(\kappa \sim 10^{-13} /(\mu \mathrm{A})^{2}\right.$ for a $100-\mathrm{MHz}$, AT cut quartz [4] ). However, higher frequency structures tend to be stiffer and need smaller gap sizes for their operation, which would mildly counteract the benefit from higher $\omega_{0}$.

\section{CONCLUSIONS}

In this work, we demonstrated that amplitude noise in the studied oscillator induces phase noise in presence of the nonlinear $A-f$ effect. A model for the $A-f$ effect was derived and both static and dynamic measurements on a double ended tuning fork resonator were presented for verification.

It was also observed that the $A-f$ effect in our MEMS test structure was much stronger than in quartz crystals. We conjecture that this disadvantage will be less pronounced in higher frequency resonators.

\section{ACKNOWLEDGEMENTS}

This work was supported by DARPA HERMIT (ONR N66001-03-1-8942), and The National Nanofabrication Users Network facilities funded by the National Science Foundation under award ECS-9731294, and The National Science Foundation Instrumentation for Materials Research Program (DMR 9504099).

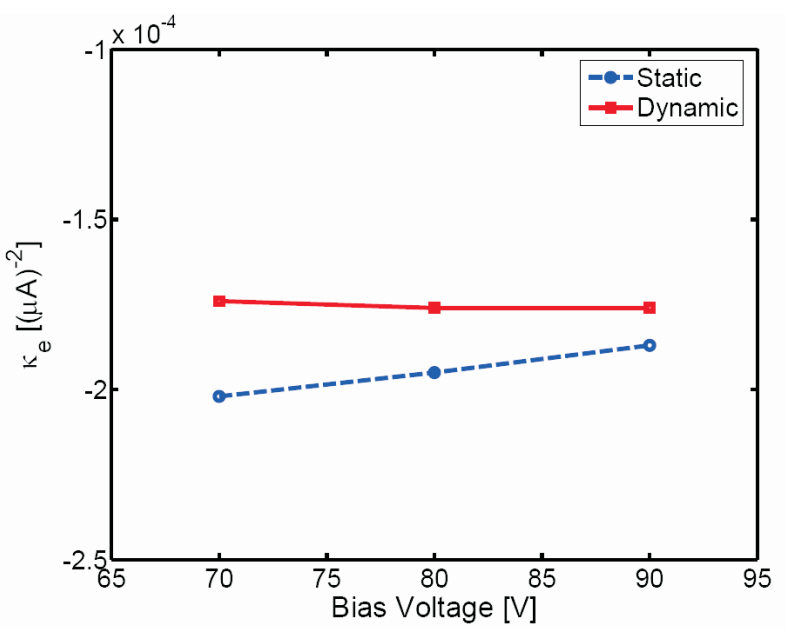

Figure 7. Comparison of $\kappa$ from different measurements.

We also thank Dr. John R. Vig, Dr. David A. Howe and Prof. David B. Leeson for their useful inputs on the subject of nonlinearities in oscillators.

\section{REFERENCES}

[1] C. T.-C. Nguyen, "Vibrating RF MEMS technology: fuel for an integrated micromechanical circuit revolution?," presented at TRANSDUCERS, 2005.

[2] Y.-W. Lin et al., "Low phase noise array-composite micromechanical wine-glass disk oscillator," presented at IEDM, 2005.

[3] V. Kaajakari et al., "Nonlinear limits for single-crystal silicon microresonators," JMEMS, vol. 13, pp. 715-724, 2004.

[4] J. J. Gagnepain, "Nonlinear Properties of Quartz Crystal and Quartz Resonators: A Review," presented at the Frequency Control Symposium, 1981.

[5] M. Agarwal et al., "Non-Linearity Cancellation in MEMS Resonators for Improved Power-Handling," presented at IEDM'05.

[6] S. Lee et al., "Influence of automatic level control on micromechanical resonator oscillator phase noise," presented at the Frequency Control Symposium, 2003.

[7] S. Lee et al., "Phase noise amplitude dependence in self limiting wine-glass disk oscillators.," presented at Hilton Head, 2004.

[8] V. Kaajakari et al., "Phase noise in capacitively coupled micromechanical oscillators," IEEE Trans. Ultrasonics, Ferroelectrics and Frequency Control, vol. 52, pp. 2322-2331, 2005.

[9] T. H. Lee, The Design of CMOS Radio Frequency Integrated Circuits, 2nd ed. New York, USA: Cambridge University Press, 2004, pp. 664-667.

[10] M. Planat et al., "Nonlinear Characteristics of SAW Grooved Resonators," presented at the Ultrasonics Symposium, 1980.

[11] L. D. Landau and E. M. Lifshitz, "Resonance in non-linear oscillations," in Mechanics, vol. 1, Course of Theoretical Physics, 3 ed. Reading, MA: Butterworth-Heinemann, 1982, pp. 87-92.

[12] R. N. Candler et al., "Hydrogen diffusion and pressure control of encapsulated MEMS resonators," presented at TRANSDUCERS, 2005.

[13] R. N. Candler et al., "Single wafer encapsulation of MEMS devices," IEEE Trans. Advanced Packaging, vol. 26, pp. 227-232, 2003.

[14] M. Planat et al., "Non-linear Propagation of Surface Acoustic Waves on Quartz," presented at Frequency Control Symposium, 1980 . 